\title{
TEXTURE-PROPERTY RELATIONSHIPS IN THE HIGH TEMPERATURE SUPERCONDUCTORS
}

\author{
A. D. ROLLETT, ${ }^{2}$ H.-R. WENK, F. HEIDELBACH, T. G. SCHOFIELD, \\ R. E. MUENSCHAUSEN, I. D. RAISTRICK, P. N. ARENDT, D. A. KORZEKWA, \\ K. BENNETT AND ${ }^{1} \mathrm{~J}$. S. KALLEND
}

Los Alamos National Laboratory, NM 87545, USA

${ }^{1}$ Illinois Institute of Technology, Chicago, IL 60616

2Univ. of California, Berkeley, CA 94720

\section{$\underline{\text { ABSTRACT }}$}

Textures have been measured by means of X-ray pole figures for high temperature superconductor materials in both bulk and thin film form. Variations in the epitaxy of the yttrium-based thin films are correlated with processing history and properties. Textures are given for deformation-processed $\mathrm{Bi}$-based material, which, when subsequently melt-processed, exhibits high critical currents. The surface resistance of Tl-based films on a silver substrate are correlated with the sharpness of the texture.

\section{INTRODUCTION}

Most of the known high temperature superconductors have anisotropic crystal structures in which the special electrical conduction properties are known to be anisotropic. Traditional texture-property studies have concentrated on making correlations with mechanical properties in metals, but for the high Tc superconductors the emphasis is naturally on correlations with electrical properties. The $\mathrm{Y}_{2} \mathrm{BaCu}_{3} \mathrm{O}_{7}$ compound ( $\left.\mathrm{Y}-123\right)$, for example, is orthorhombic, albeit close to tetragonal, and the superconduction occurs on the a-b plane. This simple fact has motivated a continuing study of the preferred orientation, texture, of samples of the compound. It also appears that grain boundaries are detrimental to superconductivity and that the strength of the barrier increases with increasing misorientation at a boundary ${ }^{1}$. This dependence on misorientation suggests that texture needs to be measured locally in order to be able to determine misorientation distributions. Nevertheless, conventional measurements of texture with X-ray pole figures can yield useful data for correlations with properties. Similar considerations apply in the Bi-based and Tl-based compounds discussed below.

A further complication for texture analysis presented by the Y-123 compound is that the c:a ratio of the lattice is close to 3 so that, for example, the (003) and (100) reflections are nearly degenerate and their pole figures overlap. If the structure factors for the individual \{hkl\} reflections are known, the orientation distribution (OD) can be calculated from overlapping pole figures, either with a WIMV algorithm ${ }^{2}$ or by the harmonic method ${ }^{2}$. Such an analysis depends on having at least one pole figure available that unambiguously defines the distribution of the c-axes. An example of such an analysis has been performed recently for an $\mathrm{Y}-123$ sample that showed a strong $<001>$ fibre texture ${ }^{3}$. 


\section{EXPERIMENTAL}

The majority of the results reported here are for thin films $(<1 \mu \mathrm{m})$ on single crystal substrates which in almost all cases were epitaxial with their substrates. The majority of the films were manufactured by co-evaporating $\mathrm{Y}, \mathrm{Cu}$ and $\mathrm{BaF}_{2}$ onto the substrate in a vacuum of $5 \times 10^{-8}$ torr, and then annealing in oxygen and water vapor ${ }^{4}, 5$. Some films were made by vapor deposition from laser ablation of the Y-123 compound onto single crystal substrates under a $20 \mathrm{~Pa}$ partial pressure of oxygen. X-ray powder diffraction analysis of the films showed small amounts of impurity phases in many cases although quantification of the impurity content was not attempted because of the high degree of preferred orientation. The T1-based films were prepared by DC magnetron sputtering a metal target with 1223 composition $(\mathrm{TlCaBaCu})$ onto various substrates. The mixed oxide was generated by heating in oxygen and $\mathrm{Tl}$ oxide for times and temperatures up to 20 minutes and $900^{\circ} \mathrm{C}$, as described previously ${ }^{6}$.

Pole figure analysis was carried out on a pole figure goniometer set for back reflection measurement. Pole figures were measured out to a tilt angle of $80^{\circ}(\chi)$ with a full $360^{\circ}$ of rotation $(\phi)$. The raw data have been corrected for defocussing 7 and background subtraction before plotting. The epitaxial thin films of superconductor present special problems because of their quasi-single crystal nature. For example, the films are thin enough $(<1 \mu \mathrm{m}$ in many cases) that diffraction from the substrate obscures the $010 / 003$ and $103 / 110$ pole figures and the defocussing correction is affected. Since the source of X-rays is not strictly monochromatic, diffraction of the part of the spectrum that is not close to the $\mathrm{K}_{\alpha}$ line leads, for example, to enhanced background counts at $\chi=0^{\circ}$ from $\{001\}$ diffraction..
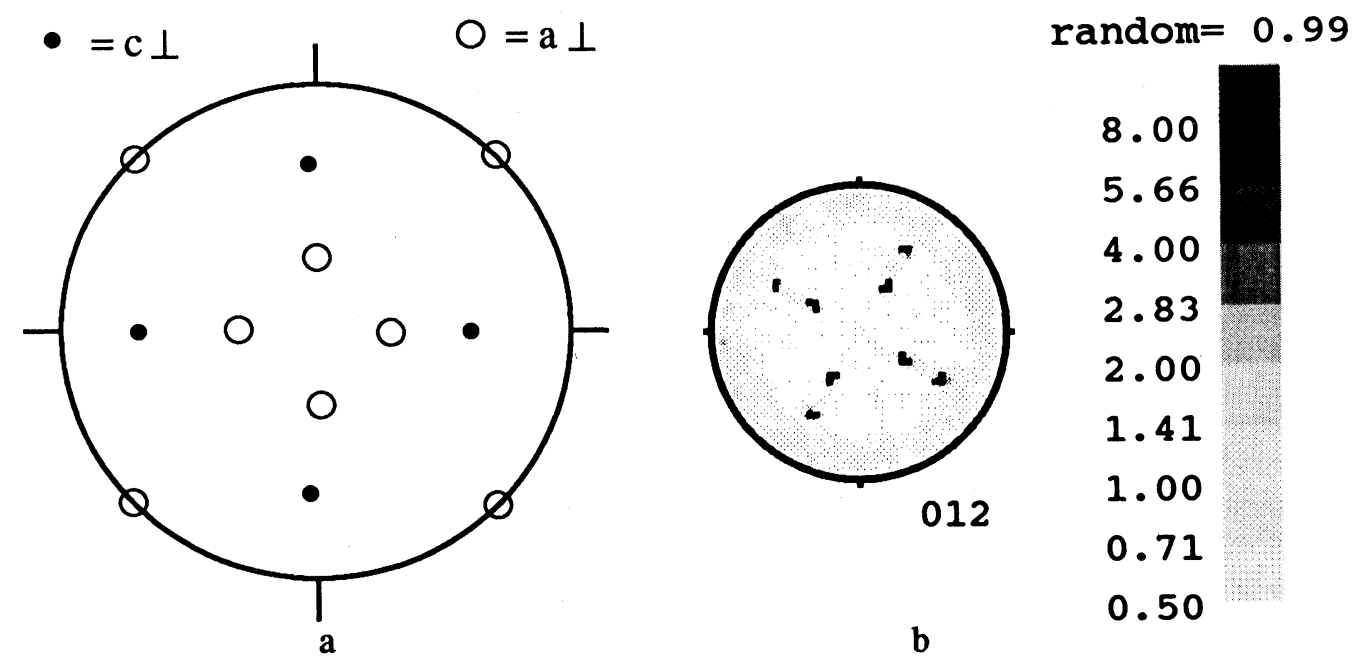

Fig. 1. a) Diagram illustrating the positions of the observable maxima in the $\{012\} /\{102\}$ pole figure. Note that other maxima for a-normal material exist at $\chi=90^{\circ}$ but cannot be observed in a reflection pole figure; b) $\{012\}$ pole figure for a Y-123 thin film on a $\mathrm{LaAlO}_{3}$ substrate, showing the eight maxima at $\chi=35^{\circ}$ and $55^{\circ}, \phi=0^{\circ}, 90^{\circ}, 180^{\circ} \& 270^{\circ}$; scale bar at left shows contour levels for the pole figure. 
The limited angular resolution of a pole figure goniometer means that the orthorhombic $Y$ 123 compound must be treated as tetragonal. The epitaxial thin films are poly-crystalline with some crystals aligned with their c-axis normal to the film ("c-normal") and some with their c-axis lying in the plane of the film ("a-normal"). The proportions of these two orientations were measured by scanning over the eight $012 / 102$ diffraction maxima at $\chi=35^{\circ}$ for a-normal material and $\chi=55^{\circ}$ for c-normal material ${ }^{8}\left(\phi=0,90,180,270^{\circ}\right)$. Owing to variations in peak shape and position, however, multiple scans were made over each of the eight maxima and the areas under each scan were integrated to arrive at a "c $\perp: a \perp$ " ratio. The limited amount of transmission electron microscopy on Y-123 superconductor has tended to confirm the X-ray results ${ }^{9}$.

\section{RESULTS}

\section{Electrophoretically Deposited Films}

Hein et al. ${ }^{10}$ have reported the production of thick films of the Y-123 compound by electrophoretic deposition from an organic suspension onto silver substrates. Figure 2 shows the $\{003\},\{012\} /\{102\}$ and $\{110\} /\{103\}$ pole figures for two films, one of which was produced in an 8T field and the other of which was produced without an applied field. The applied magnetic field clearly produced a more highly textured film that had correspondingly reduced surface resistance $(2 \mathrm{~m} \Omega)$ in an $\mathrm{rf}$ test in a $21.5 \mathrm{GHz}$ microwave cavity (compare with $18 \mathrm{~m} \Omega$ for the less aligned film). The superconducting transition temperature was also higher $(\sim 90 \mathrm{~K})$ for the more textured film than for the zero-magnetic field film $(\sim 87 \mathrm{~K})$. Table 1 compares the intensities (as a multiple of a random intensity) at the center of each pole figure and the results show quantitatively that the film with the lower resistivity was more strongly aligned.

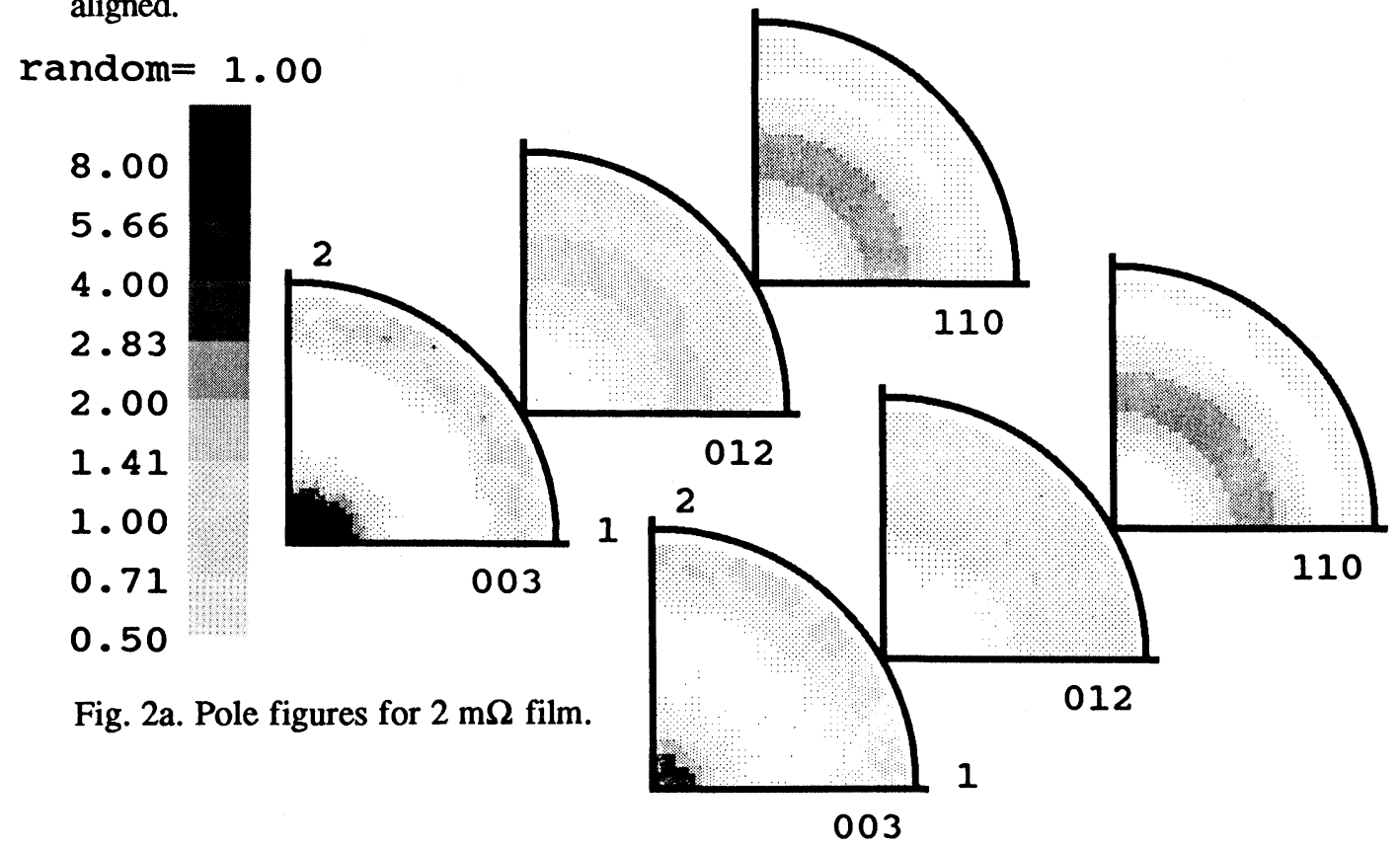

Fig. 2b. Pole figures for $18 \mathrm{~m} \Omega$ Y -123 film. 
Table 1. Intensity (times random) at the center of each pole figure (but at $\chi=55^{\circ}$ for $102 / 012$ ) for two electrophoretically deposited films of $Y-123$. The $2 \mathrm{~m} \Omega$ film was produced in an $8 \mathrm{~T}$ field.

\begin{tabular}{|c|c|c|}
\hline Reflection & $2 \mathrm{~m} \Omega$ film & $18 \mathrm{~m} \Omega$ film \\
\hline \hline 001 & 6.6 & 2.2 \\
\hline 002 & 15 & 5.1 \\
\hline $003 / 100 / 010$ & 18 & 3.5 \\
\hline $012 / 102$ & 2 & 1.3 \\
\hline
\end{tabular}

\section{Co-evaporated Films}

Table 2. Variation of Alignment of $\mathrm{Y}_{2} \mathrm{BaCu}_{3} \mathrm{O}_{7}$ Films with Processing History.

\begin{tabular}{|c|c|c|c|}
\hline Temperature & time (hours) & $\mathrm{c} \perp: \mathrm{a} \perp$ ratio & FWHM \\
\hline $750^{\circ} \mathrm{C}$ & 2 & $3.5: 1$ & 3.5 \\
\hline $750^{\circ} \mathrm{C}$ & 6 & $21: 1$ & 2.9 \\
\hline $750^{\circ} \mathrm{C}$ & 18 & $11: 1$ & - \\
\hline $800^{\circ} \mathrm{C}$ & 1 & $30: 1$ & 3.75 \\
\hline $800^{\circ} \mathrm{C}$ & 2 & $6: 1$ & 4 \\
\hline $800^{\circ} \mathrm{C}$ & 6 & $22.5: 1$ & 3.5 \\
\hline $800^{\circ} \mathrm{C}$ & 18 & $32: 1$ & 4 \\
\hline $850^{\circ} \mathrm{C}$ & 18 & $119: 1$ & 3.25 \\
\hline
\end{tabular}

For films produced by the co-evaporation technique outlined above, numerous measurements of texture have been made. There is a clear trend in the texture of the films as the annealing treatments are varied, with higher temperatures giving higher $c-\perp: a-\perp$ ratios, see Table 2 . The variation with time is less clear though the $800^{\circ} \mathrm{C}$ results suggest a tendency for increasing c$\perp: a-\perp$ ratio with time. The lack of variation of the width of the diffraction maxima indicates that the mosaic spread in these films is much smaller than can be measured by a pole figure goniometer. Long annealing times, however, lead to significant interdiffusion of the substrate and superconductor so films were made with short, high-temperature annealing treatments. The results of the comparison of short and long annealing times are shown in Table 3.

Table 3. Properties and Processing History for $0.22 \mu \mathrm{m}$ thick $\mathrm{Y}_{2} \mathrm{BaCu}_{3} \mathrm{O}_{7}$ Films.

\begin{tabular}{|l|l|l|l|l|}
\hline Annealing & $\begin{array}{l}\text { Resistivity } \\
100 \mathrm{~K}(\Omega)\end{array}$ & $\mathrm{c} \perp: \mathrm{a} \perp$ ratio & $\begin{array}{l}\text { Intensity in 002 } \\
\mathrm{PF}(\mathrm{x} \text { random })\end{array}$ & $\begin{array}{l}\text { RBS channeling } \\
\text { yield }(\%)\end{array}$ \\
\hline $10 \mathrm{~min}, 850^{\circ} \mathrm{C}$ & 1.5 & 339 & 19 & 17 \\
\hline $3 \mathrm{hrs}, 850^{\circ} \mathrm{C}$ & 3.1 & 727 & 71 & 32 \\
\hline $10 \mathrm{~min}, 900^{\circ} \mathrm{C}$ & 1.85 & 560 & 189 & 7 \\
\hline $3 \mathrm{hrs}, 900^{\circ} \mathrm{C}$ & 2.15 & 538 & 172 & 11 \\
\hline
\end{tabular}


The normal state resistivity is generally a good indicator of the quality of the film and in this case it appears to be positively correlated with the Rutherford Backscattering (RBS) yield (percentage of random material) which is a measure of the crystallographic perfection of the film. Comparing different annealing times at the same temperature, higher RBS yields correlate with higher resistivities at $100 \mathrm{~K}$. The intensity at the center of the $(002)$ pole figure is also a measure of the spread in orientation of the film and this appears to show the same correlation at $900^{\circ} \mathrm{C}$ in the sense that the longer annealing time gives a lower intensity. The $c-\perp: a-\perp$ ratio also decreases slightly with annealing time. This correlation does not hold at the lower temperature, however. The conclusion is that the electrical properties are correlated with crystallographic quality, albeit not consistently.

\section{Laser-Sputtered Films}

A large number of films have been prepared by laser-sputtering of the superconducting compound itself onto single crystal substrates ${ }^{11}$. In the series of thin films reported here, the process variable is the temperature at which the films are deposited. Figure 3 plots the variation of $c-\perp: a-\perp$ ratio with reciprocal deposition temperature. The results show that the amount of c-normal material decreases with deposition temperature in a manner that suggests that an Arrhenius relationship holds. Other, preliminary results suggest that there is a monotonic decrease in the angular spread as the deposition temperature is increased. These results will be discussed in more detail in a subsequent publication.

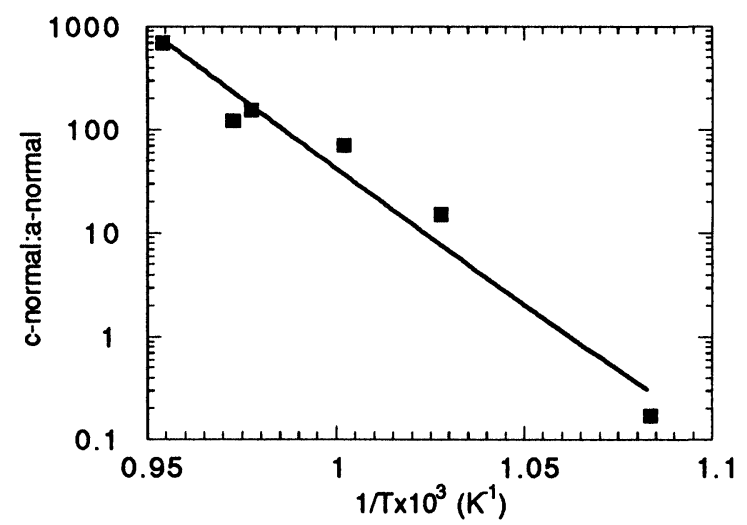

Fig. 3. Semi-logarithmic plot of $c-\perp: a-\perp$ ratio versus reciprocal deposition temperature.

\section{Bulk Samples}

The Yttrium-compound in bulk form has excited less interest than the thin-film form because it has been difficult to obtain high critical current densities though strong textures have been observed in axially compressed samples ${ }^{12}$. For the Bi-based compounds, however, good values for the critical current have been obtained recently 13 . The fabrication route involves encapsulating the 2212 compound in silver and then rolling and/or drawing the material to a tape with sub-millimeter thickness. The tape is then heated to a temperature between the melting point of the superconductor and the silver. The resulting composite can attain critical 
currents above $10^{4}$ A.cm-2 . Confined rolling of powder of the 2212 compound at room temperature in a stainless steel jacket was performed at Los Alamos. The result was a powder compact with sufficient mechanical strength that pieces could be cut with a laser for pole figure analysis. Figure 4 shows the recalculated $\{011\},\{113\}$ and $\{008\}$ pole figures obtained by orientation distribution analysis using the WIMV analysis ${ }^{2}$ for the corresponding experimental pole figures. The pole figures show that a pronounced texture is developed with an orthorhombic sample symmetry as expected for plane-strain compression. Neutron pole figures have also been measured for this material at LANSCE using the time-of-flight neutron source; this work will be reported in a later publication but the preliminary results confirm the $\mathrm{X}$-ray results.

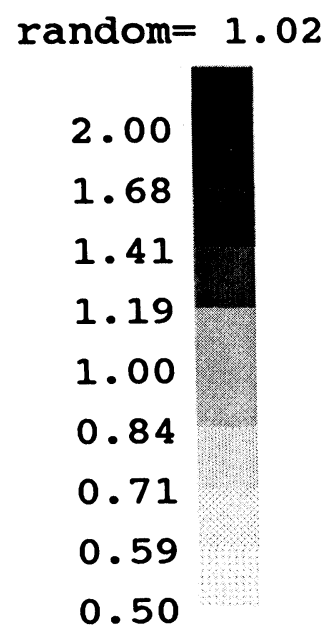

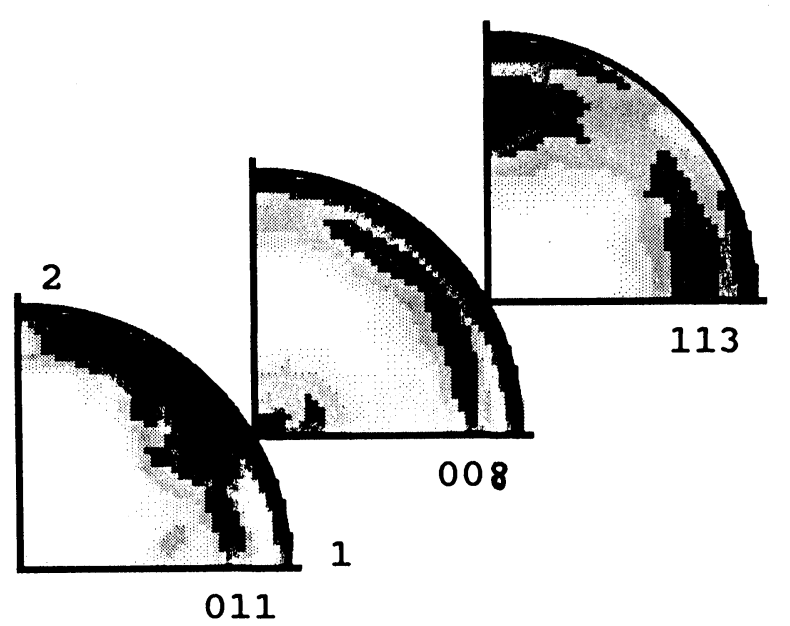

b
C

Fig. 4. Pole figures for cold-rolled 2212 Bi-based superconductor recalculated from the orientation distribution; a) $\{011\}$ pole figure, b) $\{008\}$ pole figure c) $\{113\}$ pole figure.

\section{Tl-based films}

The texture of three Tl-based films were measured and the $\{102\}$ pole figures for the 1212 compound are compared in Fig. 5. The films were approximately $11 \mu \mathrm{m}$ thick, on $38 \mathrm{~mm}$ diameter substrates of Consil 995 . One of the films (\#3) was predominantly composed of the 1212 compound but the other two films also had significant amounts of 2212 . The pole figures for the 2212 component indicated that the character and sharpness of texture was similar for the 2212 component as for the 1212 . The character of the textures is clearly a caxis fiber, which is a desirable aligment but not as good as the epitaxial alignment observed for single crystal substrates, which is reflected in the surface resistivity as a function of temperature. The surface resistivity was measured as a function of magnetic field at $4 \mathrm{~K}$, Fig. 6 , from which it is clear that superconducting behavior is less sensitive to magnetic field as the texture sharpens. The sharpness of the texture can be quantified in several ways but, for 
example, the intensity (multiples of random) at the center of the 002 pole figure varied as 15 (\#1), 48 (\#2) and 170 (\#3).

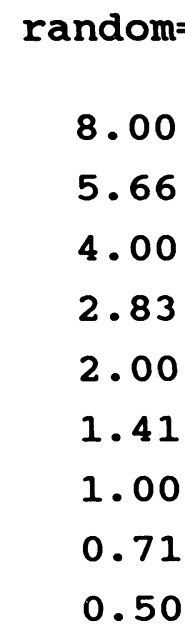

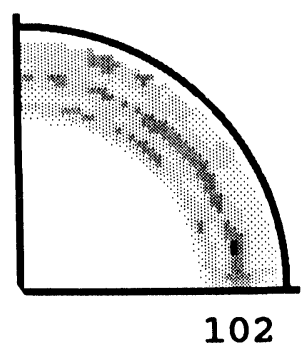

a

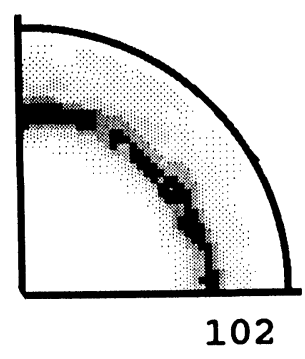

b

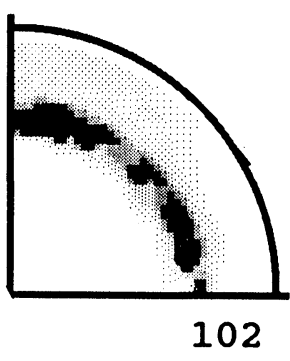

c

Fig. 5. $\{102\}$ pole figures for three films of Tl-based superconductor on silver substrates, a) $\# 1$, b) \#2, c) \#3. Note that the textures are predominantly $<001>$ fiber textures.

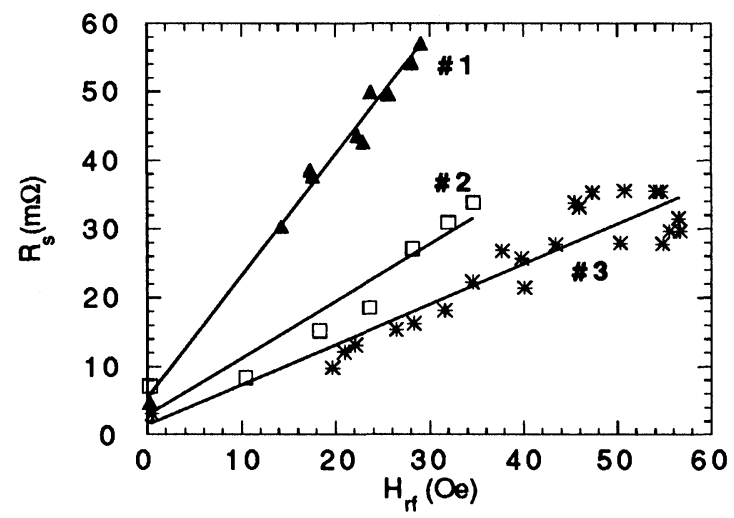

Fig. 6. Plot of $R_{S}$ versus applied magnetic field for the three films of Tl-based superconductor on Ag substrates, measured at $18 \mathrm{GHz}$ and $4 \mathrm{~K}$. 


\section{DISCUSSION AND CONCLUSIONS}

It is clear from the results presented above that changes in texture are often correlated with variations in the electrical properties of the high-temperature superconductors, as expected from their anisotropic crystal structures. So, for example, in the case of T1-based films, the sharper the fiber texture, the better the films perform in a magnetic field. So far then, the texture studies have been successful though much more so in defining the effect of processing history on texture. What is still missing, however, is a quantitative theory to connect some measure of texture and the electrical properties of the material in the manner of the well established methods for calculating anisotropic elastic and plastic properties from orientation distributions.

\section{ACKNOWLEDGEMENTS}

The support of OBES is acknowledged for ADR, INCOR for HRW and KB, and LANSCE for FH.

\section{REFERENCES}

1. T. R. Dinger, T. W. Worthington, W. J. Gallagher and R. L. Sandstrom, Phys. Rev. Lett., 58, 1676 (1987).

2. J. S. Kallend, U. F. Kocks, A. D. Rollett and H.-R. Wenk,"Operational Texture Analysis", Mat. Sci. \& Eng., accepted for publ.

3. J. S. Kallend, R. Schwarz and A. D. Rollett, submitted to Textures and Microstructures.

4. P. M. Mankiewich, J. H. Schofield, W. J. Skocpol, R. E. Howard, A. H. Dayem and E. Good, Appl. Phys. Lett., 51, 1753 (1987).

5. F. H. Garzon, J. G. Beery, D. R. Brown, R. J. Sherman and I. D. Raistrick, Appl. Phys. Lett., 54, 1365 (1989).

6. P. Arendt, W. Bongiani, N, Elliott and R. E. Muenchausen, in Science and Technology of Thin Film Superconductors, ed. R. D. McConnell and S. A. Wolf, pp. 185, Plenum, New York (1989).

7. R. E. Bolmaro, in preparation.

8. J. Sizemore, R. Barton, A. Marshall, J. C. Bravman, M. Naito and K. Char, IEEE Trans. Magnetics, 25, 2245 (1989).

9. T. Roy, I. D. Raistrick and T. E. Mitchell, Proc. Materials Res. Soc., 169 (1989); T. Roy and T. E. Mitchell, Proc. 12th Int. Congr. Elec. Microscopy, San Francisco Press, 42 (1990).

10. M. Hein, G. Müller, H. Piel, L. Ponto, M. Becks, U. Klein and M. Peiniger, J. Appl. Phys., 66, 5940 (1989).

11. R. E. Muenchhausen, K. M. Hubbard, S. Foltyn, R. C. Estler, N. S. Nogar, C. Jenkins, Appl. Phys. Lett., 56, 578 (1990).

12. H.-R. Wenk, J. Pannetier, G. Bussod and A. Pechenik, J. Appl. Phys., 65, 4070 (1989).

13. K. Heine, J. Tenbrink and M. Thöner, Appl. Phys. Lett., 55, 2441 (1989). 